\title{
PERIODIC STRUCTURE OF TRANSVERSAL MAPS ON SUM-FREE PRODUCTS OF SPHERES
}

\author{
JAUME LLIBRE ${ }^{1}$ AND VÍCTOR F. SIRVENT ${ }^{2}$
}

\begin{abstract}
In this article we study the periodic structure of transversal maps on the product of spheres of different dimensions. In particular we give conditions for the maps to have infinitely many even and odd periods. Moreover we give conditions for having non-zero Lefschetz numbers of period $m$, for infinitely many $m$ 's. We generalize the results for transversal maps on rational exterior spaces of rank 1 .
\end{abstract}

\section{INTRODUCTION}

Let $X$ be a $n$-dimensional topological manifold and $f$ a continuous self-map on $X$. The map $f$ induces a homomorphism on the $k$-th rational homology group of $X$ for $0 \leq k \leq n$, i.e. $f_{* k}: H_{k}(X, \mathbb{Q}) \rightarrow$ $H_{k}(X, \mathbb{Q})$. The $H_{k}(X, \mathbb{Q})$ is a finite dimensional vector space over $\mathbb{Q}$ and $f_{* k}$ is a linear map whose matrix has integer entries.

The Lefschetz number of $f$ is an integer defined as

$$
L(f)=\sum_{k=0}^{n}(-1)^{k} \operatorname{trace}\left(f_{* k}\right) .
$$

The Lefschetz Fixed Point Theorem states that if $L(f) \neq 0$ then $f$ has a fixed point ( $c f .[2]$ or [9]).

The Lefschetz numbers of period $m$ are defined by

$$
\ell\left(f^{m}\right):=\sum_{r \mid m} \mu(r) L\left(f^{m / r}\right)
$$

Date: July 29, 2018.

2010 Mathematics Subject Classification. 37C25,37C30,37E15.

Key words and phrases. Transversal maps, Lefschetz numbers, periodic point, product of spheres. 
where the sum is taken over all divisors $r$ of $m$ and $\mu$ is the Möbius function defined by

$$
\mu(m):= \begin{cases}1 & \text { if } m=1 ; \\ 0 & \text { if there is a } k \text { such that } k^{2} \text { divides } m \\ (-1)^{s} & \text { if } m=p_{1} \cdots p_{s} \text { with } p_{i} \text { distinct primes. }\end{cases}
$$

By the Möbius inversion formula

$$
L\left(f^{m}\right)=\sum_{r \mid m} \ell\left(f^{r}\right)
$$

In this article we consider the product of spheres with different dimensions, i.e. $X=\mathbb{S}^{n_{1}} \times \cdots \times \mathbb{S}^{n_{k}}$, with $n_{1}<\cdots<n_{k}$. Let $M$ be the set of sums of all the subsets of the numbers $n_{1}, \ldots, n_{k}$, i.e.

$$
M=M\left(n_{1}, \ldots, n_{k}\right):=\bigcup_{s=1}^{k}\left\{n_{i_{1}}+\cdots+n_{i_{s}}: i_{1}<\cdots<i_{s}\right\} .
$$

By elementary combinatorics we have that the cardinality of the set $M$ is at most $2^{k}-1$. We shall assume that the cardinality of $M$ is exactly $2^{k}-1$, i.e. the numbers $n_{1}, \ldots, n_{k}$ are such that all the sums defined in the set $M$ are different. In this case we say that $M$ is a sum free set. In this situation, by the Künneth Theorem ( $c f$. [7]), we get that the Betti numbers of $X$ are $b_{j}=1$ if $j \in M$ and $b_{j}=0$ otherwise, i.e.

$$
H_{j}(X, \mathbb{Q})=\mathbb{Q} \text { if } j \in\{0\} \bigcup_{s=1}^{k}\left\{n_{i_{1}}+\cdots+n_{i_{s}} \mid i_{1}<\cdots<i_{s}\right\},
$$

and trivial otherwise.

Let $f: X \rightarrow X$ be a continuous map, since the homology groups are either 1-dimensional or trivial, its induced maps on homology are $f_{* j}=\left(a_{j}\right)$ if $j \in M, f_{* 0}=(1)$ and $f_{* j}=0$ otherwise; where the numbers $a_{j}$ are integers. A study of the periodic structure of a class of self-maps on $X$, was done in [12]. So the Lefschetz numbers are

$$
\begin{aligned}
L\left(f^{m}\right) & =\sum_{j \geq 0}(-1)^{j} \operatorname{trace}\left(f_{* j}^{m}\right) \\
& =1+\sum_{j \in M}(-1)^{j} a_{j}^{m} .
\end{aligned}
$$


In this case the Lefschetz numbers of period $m$ are given by:

$$
\begin{aligned}
\ell\left(f^{m}\right) & =\sum_{r \mid m} \mu(r) L\left(f^{m / r}\right) \\
& =\sum_{r \mid m} \mu(r)\left(1+\sum_{j \in M}(-1)^{j} a_{j}^{m / r}\right) .
\end{aligned}
$$

Since $\sum_{r \mid m} \mu(r)=0$, if $m>1$ (cf. [1]), we have

$$
\begin{aligned}
\ell\left(f^{m}\right) & =\sum_{j \in M}(-1)^{j}\left(\sum_{r \mid m} \mu(r) a_{j}^{m / r}\right) \\
& =\sum_{j \in M}(-1)^{j} Q_{m}\left(a_{j}\right),
\end{aligned}
$$

where the polynomial $Q_{m}(x)$ is defined as

$$
Q_{m}(x):=\sum_{r \mid m} \mu(r) x^{m / r} .
$$

A transversal map $f$ on a compact differentiable manifold $X$ is a $C^{1}$ map $f: X \rightarrow X$, such that $f(X) \subset \operatorname{Int}(\mathrm{X})$ and for all positive integer $m$ at each point $x$ fixed by $f^{m}$ we have that 1 is not an eigenvalue of $D f^{m}(x)$, i.e. $\operatorname{det}\left(I d-D f^{m}(x)\right) \neq 0$. The following theorem is one of the main results in relation to the periodic structure of transversal maps in the general setting.

Theorem 1 ([10,6]). Let $X$ be a compact manifold and $f: X \rightarrow X$ be a transversal map. Suppose $\ell\left(f^{m}\right) \neq 0$, for some $m$. Then

(1) If $m$ is odd then $m \in \operatorname{Per}(\mathrm{f})$.

(2) If $m$ is even then either $m$ or $m / 2$ is in $\operatorname{Per}(\mathrm{f})$.

The periodic structure of transversal maps on different context was studied in $[4,6,11]$. In the following we list our main results for transversal maps on the product of spheres. Their proofs are in section 2.

Theorem 2. Let $X=\mathbb{S}^{n_{1}} \times \cdots \times \mathbb{S}^{n_{k}}$, with $n_{1}<\cdots<n_{k}$. We assume that the set $M$ of partial sums of the dimensions of the spheres is a sum free set. Let $f$ be a transversal self-map on $X$, with $f_{* j}=\left(a_{j}\right)$ for $j \in M$, with $\left|a_{n_{i}}\right|>1$ for $1 \leq i \leq k$. Then $\ell\left(f^{m}\right) \neq 0$ with $m \geq N$, for some positive integer $N$.

From Theorems 1 and 2 we get the following corollary. 
Corollary 3. Under the assumptions of Theorem 2 there exists an integer $N$ such that

(1) If $m>N$ and odd then $m \in \operatorname{Per}(\mathrm{f})$.

(2) If $m>N$ and even then either $m$ or $m / 2$ is in $\operatorname{Per}(\mathrm{f})$.

In the following theorem we weaken the hypothesis of Theorem 2, allowing that some of the $a_{j}= \pm 1$. However we need to introduce some extra notation, which is given in the statement.

Theorem 4. Let $X=\mathbb{S}^{n_{1}} \times \cdots \times \mathbb{S}^{n_{k}}$, with $n_{1}<\cdots<n_{k}$, and $f$ be a transversal self-map on $X$, with $f_{* j}=\left(a_{j}\right)$ for $j \in M$ there is an $1 \leq i \leq k$ such that $\left|a_{n_{i}}\right|>1$. Assume that $M$, the set of partial sum of the indexes $n_{1}, \ldots, n_{k}$, is a sum-free set. Let

$$
M^{\prime}:=\left\{j^{\prime} \in M: a_{j^{\prime}}=\max _{j \in M}\left\{\left|a_{j}\right|\right\}\right\} .
$$

(a) In the case of $a_{i}=1$ whenever $\left|a_{i}\right|=1$. If $\sum_{j \in M^{\prime}}(-1)^{j} \neq 0$ then $\ell\left(f^{m}\right) \neq 0$ with $m \geq N$ for some positive integer $N$.

(b) Let $M^{\prime}=M_{1}^{\prime} \cup M_{2}^{\prime}$, where $a_{j}>0$ if $j \in M_{1}^{\prime}$, and $a_{j}<0$ if $j \in M_{2}^{\prime}$. If

$$
\sum_{j \in M_{1}^{\prime}}(-1)^{j} \pm\left(\sum_{j \in M_{2}^{\prime}}(-1)^{j}\right) \neq 0
$$

then $\ell\left(f^{m}\right) \neq 0$ with $m \geq N$ for some positive integer $N$.

(c) If $M_{1}^{\prime}$ and $M_{2}^{\prime}$ are not empty and

$$
\sum_{j \in M_{1}^{\prime}}(-1)^{j}-\left(\sum_{j \in M_{2}^{\prime}}(-1)^{j}\right)=0 \text { or } \sum_{j \in M_{1}^{\prime}}(-1)^{j}+\left(\sum_{j \in M_{2}^{\prime}}(-1)^{j}\right)=0 \text {. }
$$

Then $\ell\left(f^{m}\right) \neq 0$ with $m \geq N$ for some positive integer $N$.

Using Theorems 4 and 1 we get the following corollary:

Corollary 5. Let $X=\mathbb{S}^{n_{1}} \times \cdots \times \mathbb{S}^{n_{k}}$, with $n_{1}<\cdots<n_{k}$. and $f$ be a transversal self-map on $X$, with $f_{* j}=\left(a_{j}\right)$ for $j \in M$, with $\left|a_{n_{i}}\right|>0$ for all $1 \leq i \leq k$ and $\left|a_{n_{i}}\right|>1$ for some $1 \leq i \leq k$. Let

$$
M^{\prime}:=\left\{j^{\prime} \in M: a_{j^{\prime}}=\max _{j \in M}\left\{\left|a_{j}\right|\right\}\right\} .
$$

(a) In the case of $a_{i} \neq 1$ for all $1 \leq i \leq k$ and $\sum_{j \in M^{\prime}}(-1)^{j} \neq 0$. Then there exists an integer $N$ such that

(1) If $m>N$ and odd then $m \in \operatorname{Per}(\mathrm{f})$. 
(b) If

(2) If $m>N$ and even then either $m$ or $m / 2$ is in $\operatorname{Per}(\mathrm{f})$.

$$
\sum_{j \in M_{1}^{\prime}}(-1)^{j} \pm\left(\sum_{j \in M_{2}^{\prime}}(-1)^{j}\right) \neq 0 .
$$

Then there exists an integer $N$ such that

(1) If $m>N$ and odd then $m \in \operatorname{Per}(\mathrm{f})$.

(2) If $m>N$ and even then either $m$ or $m / 2$ is in $\operatorname{Per}(\mathrm{f})$.

Moreover if

$$
\sum_{j \in M_{1}^{\prime}}(-1)^{j}-\left(\sum_{j \in M_{2}^{\prime}}(-1)^{j}\right)=0 .
$$

Then there are infinitely many odd or even periods.

Corollaries 3 and 5-(a)-(b) state that under the hypotheses mentioned there are infinitely many even and odd periods, moreover, all odd numbers greater than $N$ are periods of the map.

In section 3 we give a generalization of Theorem 2 and Corollary 3 to rational spaces of rank 1 , which is stated in Corollary 10.

\section{Proofs of The RESUlts}

Before proving Theorem 2, we need to introduce and prove some preliminary results. Throughout this section we set $X=\mathbb{S}^{n_{1}} \times \cdots \times \mathbb{S}^{n_{k}}$, with $n_{1}<\cdots<n_{k}$, and $M$ the set of partial sums of $n_{1}, \ldots, n_{k}$.

Proposition 6. If $k \in M$, with $k=n_{i_{1}}+\cdots+n_{i_{s}}$, then $a_{k}=a_{n_{1}} \cdots a_{n_{s}}$.

Proof. Let $f_{*\left(n_{i_{1}}+n_{i_{2}}\right)}: H_{n_{i_{1}+n_{i_{2}}}}(X, \mathbb{Q}) \rightarrow H_{n_{i_{1}}+n_{i_{2}}}(X, \mathbb{Q})$ be the induced map of $f$ in the corresponding homology space. Since $H_{n_{i_{1}}+n_{i_{2}}}(X, \mathbb{Q})=$ $H_{n_{i_{1}}}(X, \mathbb{Q}) \otimes H_{n_{i_{2}}}(X, \mathbb{Q})$, we have

$$
f_{*\left(n_{i_{1}}+n_{i_{2}}\right)}\left(x_{1} \wedge x_{2}\right)=a_{n_{i_{1}+n_{i_{2}}}}\left(x_{1} \wedge x_{2}\right),
$$

where $x_{j} \in H_{n_{i_{j}}}$. On the other hand, using the properties of the wedge product:

$$
\begin{aligned}
f_{*\left(n_{i_{1}}+n_{i_{2}}\right)}\left(x_{1} \wedge x_{2}\right) & =f_{* n_{i_{1}}}\left(x_{1}\right) \wedge f_{* n_{i_{2}}}\left(x_{2}\right)=\left(a_{n_{i_{1}}} x_{1}\right) \wedge\left(a_{n_{i_{2}}} x_{2}\right) \\
& =\left(a_{n_{i_{1}}} a_{n_{i_{2}}}\right) x_{1} \wedge x_{2} .
\end{aligned}
$$

Hence $a_{n_{i_{1}}+n_{i_{2}}}=a_{n_{i_{1}}} a_{n_{i_{2}}}$. The statement follows by induction on the index $s$. 
Proposition 7. Let $\left\{P_{m}(x)\right\}_{m \geq 1}$ be a family of polynomials with bounded coefficients indexed by their degree. Given $\beta \in \mathbb{C}$, with $|\beta|>1$. Then there exist a positive integer $N$ and positive constants $C_{\beta}, D_{\beta}$ such that

$$
C_{\beta}|\beta|^{m} \leq\left|P_{m}(\beta)\right| \leq D_{\beta}|\beta|^{m}, \text { for } m \geq N \text {. }
$$

If $|\beta|=1$ then there exists a positive constant $C_{\beta}^{\prime}$

$$
\left|P_{m}(\beta)\right| \leq C_{\beta}^{\prime} m .
$$

Proof. Let

$$
P_{m}(x)=c_{m, m} x^{m}+\cdots+c_{m, 1} x+c_{m, 0},
$$

with $c_{m, m} \neq 0$ and $\left|c_{m, j}\right| \leq K$ for all $m>0$ and $0 \leq j \leq m$ and some $K$ independent from $m$.

Given $\beta$, with $|\beta|>1$, we can write

$$
\left|P_{m}(\beta)\right|=|\beta|^{m}\left|c_{m, m}+\frac{c_{m, m-1}}{\beta}+\cdots+\frac{c_{m, 0}}{\beta^{m}}\right| .
$$

It is quite clear that there exist a positive integer $N$ and positive constants $C_{\beta}$ and $D_{\beta}$, satisfying (8). We remark that the values of $N, C_{\beta}, D_{\beta}$ depends only of $\beta$ and $K$.

If $|\beta|=1$ taking $C_{\beta}^{\prime}=K$ the inequality (9) holds.

Proposition 8. Let $\beta_{1}, \cdots, \beta_{r}$ be complex numbers such that $\left|\beta_{i}\right| \geq 1$ and

$$
\left|\beta_{r}\right|>\max \left\{1,\left|\beta_{1}\right|, \ldots,\left|\beta_{r-1}\right|\right\} .
$$

Let $\left\{P_{m}(x)\right\}_{m \geq 1}$ be a family of polynomials indexed by their degree. Let

$$
A_{m}:=\sum_{i=1}^{r} d_{i} P_{m}\left(x_{i}\right),
$$

for some $d_{i}$ 's. Then there exists $N$, which only depends on the $\beta_{i}$ 's and the bound of the coefficients of the polynomial family, such that $\left|A_{m}\right|>0$, for all $m \geq N$.

Proof. Using the triangular inequality we can write

$$
\left|A_{m}\right| \geq\left|d_{r} P_{m}\left(\beta_{r}\right)\right|-\left|d_{1} P_{m}\left(\beta_{1}\right)\right|-\cdots-\left|d_{r-1} P_{m}\left(\beta_{r-1}\right)\right| .
$$

If we assume that $\left|\beta_{i}\right|>1$ for $1 \leq i \leq r$, and apply Proposition 7 , for each $\beta_{i}$, There exists a positive integer $N_{1}$ that if $m>N_{1}$

$$
\begin{aligned}
\left|A_{m}\right| & \geq D_{r}\left|d_{r}\right|\left|\beta_{r}\right|^{m}-C_{1}\left|d_{1}\right|\left|\beta_{1}\right|^{m}-\cdots-C_{r-1}\left|d_{r-1}\right|\left|\beta_{r-1}\right|^{m} \\
& =\left|\beta_{r}\right|^{m}\left(D_{r}\left|d_{r}\right|-C_{1}\left|d_{1}\right|\left|\frac{\beta_{1}}{\beta_{r}}\right|^{m}-\cdots-C_{r-1}\left|d_{r-1}\right|\left|\frac{\beta_{r-1}}{\beta_{r}}\right|^{m}\right) .
\end{aligned}
$$


Since $\left|\beta_{i}\right|<\left|\beta_{r}\right|$, for $1 \leq i \leq r-1$, there exists $N_{2}$ such that if $m>N_{2}$ then $\left|A_{m}\right|>0$.

If we suppose that one $\beta_{i}$ has norm equal 1 , say $\left|\beta_{r-1}\right|=1$. Then, it follows from (9):

$$
\begin{aligned}
\left|A_{m}\right| \geq & D_{r}\left|d_{r}\right|\left|\beta_{r}\right|^{m}-C_{1}\left|d_{1}\right|\left|\beta_{1}\right|^{m}-\cdots C_{r-2}\left|d_{r-2}\right|\left|\beta_{r-2}\right|^{m}-C_{r-1}^{\prime} m \\
= & |\beta|^{m}\left(D_{r}\left|d_{r}\right|-C_{1}\left|d_{1}\right|\left|\frac{\beta_{1}}{\beta_{r}}\right|^{m}-\cdots-\right. \\
& \left.-C_{r-2}\left|d_{r-2}\right|\left|\frac{\beta_{r-2}}{\beta_{r}}\right|^{m}-C_{r-1}^{\prime}\left|d_{r-1}\right| \frac{m}{\left|\beta_{r}\right|^{m}}\right) .
\end{aligned}
$$

Hence there exists $N_{3}$ such that if $m>N_{3}$ then $\left|A_{m}\right|>0$.

Note the previous argument can be extended when there are at most $r-1, \beta_{i}$ 's with $\left|\beta_{i}\right|=1$.

In the previous proposition we assume the general case that the coefficients of the polynomials $P_{m}(x)$ are complex, the numbers $\beta_{i}$ and $d_{i}$ are complex. However in the applications that we use through out the article, the numbers $\beta_{i}$ are integers and the coefficients of $P_{m}(x)$ as well as the numbers $d_{i}$ 's are in the set $\{0,1,-1\}$.

Proposition 9. Let $\beta_{1}, \cdots, \beta_{r}$ be real numbers such that $\left|\beta_{i}\right| \geq 1$ and

$$
\left|\beta_{l}\right|=\cdots=\left|\beta_{r}\right|>\max \left\{1,\left|\beta_{1}\right|, \ldots,\left|\beta_{l-1}\right|\right\} .
$$

Let $\left\{P_{m}(x)\right\}_{m \geq 1}$ be a family of polynomials with bounded coefficients and indexed by their degree. Let

$$
A_{m}:=\sum_{i=1}^{r} d_{i} P_{m}\left(x_{i}\right),
$$

for some $d_{i}$ 's.

(a) If $\beta_{l}=\cdots=\beta_{r}$ and $d_{l}+\cdots+d_{r} \neq 0$ then there exists a positive integer $N_{1}$ such that $\left|A_{m}\right|>0$ for $m \geq N_{1}$.

(b) If $\beta_{l}=\cdots=\beta_{s-1}=-\beta_{s}=\cdots=-\beta_{r}$ and $d_{l}+\cdots+d_{s-1}+$ $(-1)^{m}\left(d_{s}+\cdots+d_{r}\right) \neq 0$, for all $m$; then there exists a positive integer $N_{2}$ such that $\left|A_{m}\right|>0$ for $m \geq N_{2}$.

(c) If $\beta_{l}=\cdots=\beta_{s-1}=-\beta_{s}=\cdots=-\beta_{r}$ and $d_{l}+\cdots+d_{s-1}-$ $\left(d_{s}+\cdots+d_{r}\right)=0$ or $d_{l}+\cdots+d_{s-1}+d_{s}+\cdots+d_{r}=0$ then there exists a positive integer $N_{3}$ such that $\left|A_{m}\right|>0$ for $m \geq N_{3}$ for $m$ odd or even. 
Proof. Using the triangular inequality we have:

$$
\left|A_{m}\right| \geq\left|S_{m}(\beta)\right|-\left|d_{1} P_{m}\left(\beta_{1}\right)\right|-\cdots-\left|d_{l-1} P_{m}\left(\beta_{l-1}\right)\right|
$$

where $S_{m}(\beta):=d_{r} P_{m}\left(\beta_{r}\right)+\cdots+d_{l} P_{m}\left(\beta_{l}\right)$.

$$
\text { If } \beta_{l}=\cdots=\beta_{r}=\beta \text { then }
$$

$$
S_{m}(\beta)=d_{r} P_{m}\left(\beta_{r}\right)+\cdots+d_{l} P_{m}\left(\beta_{l}\right)=P_{m}(\beta)\left(d_{r}+\cdots+d_{l}\right) .
$$

By Proposition 7 , if $d_{r}+\cdots+d_{l} \neq 0$, then there exists positive constants $D>0, C_{1}, \ldots, C_{l-1}$ such that

$$
\left|A_{m}\right| \geq|\beta|^{m} D\left|d_{r}+\cdots+d_{l}\right|-C_{1}\left|d_{1}\right|\left|\beta_{1}\right|^{m}-\cdots-C_{l-1}\left|d_{l-1}\right|\left|\beta_{l-1}\right|^{m} .
$$

Using similar computations as in the proof of Proposition 9, we conclude statement (a).

$$
\begin{aligned}
\text { If } \beta_{l} & =\cdots=\beta_{s-1}=-\beta_{s}=\cdots=-\beta_{r}=-\beta \text {, then } \\
S_{m}(\beta) & =d_{l} P_{m}\left(\beta_{l}\right)+\cdots+d_{s-1} P_{m}\left(\beta_{s-1}\right)-d_{s} P_{m}\left(\beta_{s}\right)-\cdots-d_{r} P_{m}\left(\beta_{r}\right) \\
& =P_{m}(\beta)\left(d_{l}+\cdots+d_{s-1}\right)+P_{m}(-\beta)\left(d_{s}+\cdots+d_{r}\right) .
\end{aligned}
$$

Therefore $S_{m}(\beta)$ is a polynomial of degree $m$ in $\beta$, and the coefficient of $\beta^{m}$ is

$$
d_{l}+\cdots+d_{s-1}+(-1)^{m} d_{s}+\cdots+d_{r} .
$$

If $d_{l}+\cdots+d_{s-1}+d_{s}+\cdots d_{r} \neq 0$ and $d_{l}+\cdots+d_{s-1}-\left(d_{s}+\cdots d_{r}\right) \neq 0$, using the same argument as in (a), we get statement (b).

Observe that $d_{l}+\cdots+d_{s-1}+(-1)^{m}\left(d_{s}+\cdots d_{r}\right)=0$, cannot occur for $m$ even and odd, unless in the trivial case. Suppose that $d_{l}+\cdots+d_{s-1}+$ $(-1)^{m}\left(d_{s}+\cdots d_{r}\right)=0$ for $m$ even, so $d_{l}+\cdots+d_{s-1}+(-1)^{m}\left(d_{s}+\cdots d_{r}\right) \neq$ 0 odd. By using the arguments of Proposition 8 , we get $\left|A_{m}\right|>0$, for arbitrary large odd $m$.

Proof of Theorem 2. If all $\left|a_{n_{i}}\right|>1$ then $\left|a_{n_{1}} \cdots a_{n_{k}}\right| \geq\left|a_{n_{i_{1}}} \cdots a_{n_{i_{s}}}\right|$, with $1 \leq s \leq k$, with equality only in the trivial case. From Proposition 6 ,

$$
\left|a_{n_{1}} \cdots a_{n_{k}}\right|=\max _{j \in M}\left\{\left|a_{j}\right|\right\}>1,
$$

and moreover if $j \in M$ and $j \neq n_{1}+\cdots+n_{k}$ then $\left|a_{j}\right|<\left|a_{n_{1}} \cdots a_{n_{k}}\right|$.

We apply Proposition 8 to $\ell\left(f^{m}\right)$, since its expression given in (6) is in the same format as $A_{n}$ in this proposition. By (7) the polynomial $Q(x)$ is of degree $m$ and its coefficients are 0 or \pm 1 . Therefore if we substitute the polynomial $Q_{m}(x)$ by $P_{m}(x)$ in Proposition 8 , we can conclude that there exists $N$ such that $\ell\left(f^{m}\right) \neq 0$, for $m \geq N$. 
Proof of Theorem 4. From Proposition 6 the number $n_{i_{1}}+\cdots+n_{i_{k}}$ is an element of $M^{\prime}$ and it has more elements if there are $\left|a_{n_{j}}\right|=1$, for some $1 \leq j \leq k$.

In the case $a_{n_{j}}=1$ whenever $\left|a_{n_{j}}\right|=1$, we have $a_{j}=a_{j^{\prime}}$ if $j, j^{\prime} \in$ $M^{\prime}$. From Proposition 9-(a) it follows that if $\sum_{j \in M^{\prime}}(-1)^{j} \neq 0$, then $\ell\left(f^{m}\right) \neq 0$, for $m$ sufficiently large. Proving statement (a).

If there are $a_{n_{j}}=-1$, for some $j$. We consider the partition of the set $M^{\prime}$ into $M_{1}^{\prime}$ and $M_{2}^{\prime}$ where $a_{j}>0$ if $j \in M_{1}^{\prime}$ and $a_{j}<0$ if $j \in M_{2}^{\prime}$. From Proposition 9(b) it follows that if

$$
\sum_{j \in M_{1}^{\prime}}(-1)^{j} \pm\left(\sum_{j \in M_{2}^{\prime}}(-1)^{j}\right) \neq 0
$$

then $\ell\left(f^{m}\right) \neq 0$, for $m$ sufficiently large. Proving statement (b).

If the sets $M_{1}^{\prime}$ and $M_{2}^{\prime}$ are not empty and

$$
\sum_{j \in M_{1}^{\prime}}(-1)^{j}-\left(\sum_{j \in M_{2}^{\prime}}(-1)^{j}\right)=0, \text { or } \sum_{j \in M_{1}^{\prime}}(-1)^{j}+\left(\sum_{j \in M_{2}^{\prime}}(-1)^{j}\right)=0 .
$$

Then $\ell\left(f^{m}\right) \neq 0$, for $m$ sufficiently large odd numbers or even numbers. Proving statement (c).

\section{RATiONAL EXTERIOR SPACES OF RANK 1}

In this section we generalize the previous results for maps on rational exterior spaces of rank 1. Those spaces were introduced by Duan in [3]. In order to define them, we need to introduce some concepts and notation, for this we follow [3] and [5].

Let $X$ be a topological space and $H^{n}(X, Q)$ is $n$-th cohomology over the rationals and $H^{*}(X, \mathbb{Q})=\oplus H^{n}(X, \mathbb{Q})$ is the cohomology algebra of $X$ over the rationals, with respect of the cup product. We say that $x \in H^{n}(X, \mathbb{Q})$ is decomposable is there is $\left(x_{j}, y_{j}\right) \in H^{p_{j}}(X, \mathbb{Q}) \times$ $H^{q_{j}}(X, \mathbb{Q})$, where $p_{j}$ and $q_{j}$ are positive integers such that $p_{j}+q_{j}=n$ and $x=\sum x_{j} \cup y_{j}$, where $\cup$ is the cup product of $H^{*}(X, \mathbb{Q})$. Let $D^{n}(X)$ be the the subspace of $H^{n}(X, \mathbb{Q})$ of all decomposable elements, $A^{n}(X)=H^{n}(X, \mathbb{Q}) / D^{n}(X)$ and $A(X)=\oplus A^{n}(X)$, which is a graded vector space over $\mathbb{Q}$. The dimension of $A(X)$ over $\mathbb{Q}$ is called the rank of $X$. 
Let $f$ be a continuous self-map on $X$, we denote by $A^{n}(f)$ the induced homeomorphism on $A^{n}(X)$ and $A(f)$ the induced graded homeomorphism on $A(X)$.

We say that $X$ is a rational exterior space if $X$ is a connected topological space with homogenous elements $x_{i} \in H^{\text {odd }}(X, \mathbb{Q})$, with $i=1, \ldots, r$ such that the inclusions $x_{i} \hookrightarrow H^{*}(X, \mathbb{Q})$ give rise to a ring isomorphism $\Lambda_{\mathbb{Q}}\left(x_{1}, \ldots, x_{r}\right) \cong H^{*}(X, Q)$, where $\Lambda_{\mathbb{Q}}\left(x_{1}, \ldots, x_{r}\right)$ is the exterior algebra generated by the $x_{i}$ 's.

All finite dimensional Lie groups are rational exterior spaces. The rank of the $n$-dimensional torus is $n$. The rank of $\mathbb{S}^{n} \times \mathbb{S}^{n}$ is 2 . The rank of the space consider in this article is 1, i.e. $X=\mathbb{S}^{n_{1}} \times \cdots \times \mathbb{S}^{n_{k}}$, with the set of partial sums of the $n_{i}$ 's being a sum free set. The following Lie groups are rational exterior spaces of rank 1: $S U(2), S U(3), S U(4)$, $S O(3), S O(5), S O(7), S p(1), S p(2)$ and $S p(3)$ (cf. [13]).

The proof of Corollary 3 can be adapted vis-a-vis for transversal maps on rational exterior spaces of rank 1, which are compact manifolds.

Corollary 10. Let $X$ be a compact manifold, which is a rational exterior space of rank 1 and $f$ be a transversal self-map on $X$, with $A^{j}(f)=\left(a_{j}\right)$, whenever $H^{j}(X, \mathbb{Q})$ is not trivial. If there exists $i$ such that $\left|a_{i}\right|>\left|a_{j}\right| \geq 1$ for all $j \neq i$. Then there exists an integer $N$ such that

(1) If $m>N$ and odd then $m \in \operatorname{Per}(\mathrm{f})$.

(2) If $m>N$ and even then either $m$ or $m / 2$ is in $\operatorname{Per}(\mathrm{f})$.

\section{ACKNOWLEDGEMENTS}

This work is supported by the Ministerio de Economía, Industria y Competitividad, Agencia Estatal de Investigación grant MTM201677278-P (FEDER), the Agència de Gestió d'Ajuts Universitaris i de Recerca grant 2017 SGR 1617, and the European project DynamicsH2020-MSCA-RISE-2017-777911.

Part of this work was done during a visit of the second author to Centre de Recerca Matèmatica (CRM), in the framework of the Lluis Santaló fellowship.

The authors would like to thank Marcos J. González for useful discussions and for pointing out Proposition 6. 


\section{REFERENCES}

[1] T.M. Apostol, Introduction to Analytic Number Theory, Springer-Verlag, New York, 1976.

[2] R.F. Brown, The Lefschetz fixed point theorem, Scott, Foresman and Company, Glenview, IL, 1971.

[3] H. Duan, The Lefschetz numbers of iterated maps. Topology Appl. 67 (1995), no. $1,71-79$.

[4] J.L. García Guirao and J. Llibre, Periodic structure of transversal maps on $\mathbb{C} P^{n}, \mathbb{H} P^{n}$ and $\mathbb{S}^{p} \times \mathbb{S}^{q}$, Qual. Theory Dyn. Syst. 12 (2013), no. 2, 417-425.

[5] G. Graff, Algebraic periods of self-maps of a rational exterior space of rank 2. Fixed Point Theory Appl. 2006, Special Issue, Art. ID 80521, 9 pp.

[6] A. Guillamón, X. Jarque, J. Llibre, J. Ortega and J. Torregrosa, Periods for transversal maps via Lefschetz numbers for periodic points. Trans. Math. Soc. 347 (1995), 4779-4806.

[7] A. Hatcher, Algebraic topology, Cambridge University Press, Cambridge (2002).

[8] J. Jezierski and W. Marzantowicz, Homotopy methods in topological fixed and periodic points theory, Springer Verlag, Berlin, 2006.

[9] S. Lefschetz, Intersections and transformations of complexes and manifolds, Trans. Amer. Math. Soc. 28 (1926), 1-49.

[10] J. Llibre, Lefschetz numbers for periodic points, Contemporary Math., vol 152 (1993), 215-227, Amer. Math. Soc., Providence.

[11] J. LliBRE A note on the set of periods of transversal homological sphere selfmaps, J. Differ. Equ. Appl. 20 (2003), 417-422.

[12] J. Llibre And V.F. Sirvent, On Lefschetz periodic point free self-maps, $J$. Fixed Point Theory Appl. 20 (2018), no. 1, Art. 38, 9 pp.

[13] H. Samelson, Topology of Lie groups, Bull. Amer. Math. Soc., 58 (1952), $2-37$.

${ }^{1}$ Departament de Matemàtiques. Universitat Autònoma de Barcelona, Bellaterra, 08193 Barcelona, Catalunya, Spain

E-mail address: jllibre@mat.uab.cat

2 Departamento de Matemáticas, Universidad Simón Bolívar, Apartado 89000, Caracas 1086-A, Venezuela

E-mail address: vsirvent@usb.ve 